\title{
The Analysis of Assortative Mating: A LISREL Model
}

\author{
Michael C. Neale ${ }^{1}$ and J. J. McArdle ${ }^{2}$
}

Received 6 Feb. 1989-Final 13 July 1989

\begin{abstract}
The use of linear models to discriminate between primary and secondary assortative mating has allowed a significant advance in our understanding of the mate selection process. We describe how these methods may be implemented in the LISREL and COSAN packages and illustrate the method with data on cognitive ability, education, and personality reported by Phillips et al. (Behav. Genet. 18:347-356, 1988). Issues discussed include the interface between path diagrams and computer program specification, the near-independence of parameters for within-person correlations from parameters for marital correlations, and the fact that almost all of marital resemblance for IQ seems to be due to assortative mating for educational level.
\end{abstract}

KEY WORDS: assortative mating; LISREL; COSAN.

\section{INTRODUCTION}

The issue of whether a marital correlation for two variables arises from direct assortment or from correlation with another variable on which assortment occurs has a long history. Pearson and Lee (1903) published within-person (phenotypic) and marital correlations of height, forearm length, and span in a large sample. Surprisingly, they showed that there was likely to be direct assortment for both height and forearm length. Recent developments in linear modeling make statistical tests of such findings possible. The concepts of the

\footnotetext{
The work described in this paper was performed while in reccipt of NIH, NIMH, and NIA Grants MH-40828, AA-06781, GM-30250, AG-04954, HL-31010, and AG-07137.

${ }^{1}$ Department of Human Genetics, Medical College of Virginia, Box 33, Richmond, Virginia 23298.

${ }^{2}$ Department of Psychology, Gilmer Hall, University of Virginia, Charlottesville, Virginia 22903.
} 
copath (Cloniger, 1980), the delta path (Van Eerdewegh, 1982), and the conditional path (Carey, 1986) permit modeling of the mate selection process which allows for the indirect effects of assortative mating. In this article, we show how the reticular action model's graphic and algebraic notation (RAM; see McArdle and McDonald, 1984, McArdle and Horn, 1989) may be applied to formulate the full expected matrix for phenotypic and marital covariances. This method is implemented using the LISREL 7 structural equation modeling package (Jöreskog and Sörbom, 1988) and the COSAN II package (Fraser, 1988) in order to make the analysis of assortative mating more readily available to other researchers.

Conditional path methods were applied by Phillips et al. (1988) to data from two domains, cognitive performance and personality. In the cognitive performance domain there were two variables, $g$-factor IQ and years of education. In the personality domain there were four personality scales extracted from Cattell's 16PF (Cattell et al., 1970), namely, Extraversion, Anxiety, Tough Poise, and Independence. Phillips and co-workers' (1988) analysis focused on the marital correlations, and the expected covariances within persons were fixed to equal the observed in order to facilitate speedy computation. Thus, they assumed that the phenotypic correlations were uncorrelated with parameters describing the mate selection process. Although this assumption seems reasonable, it was neither necessary nor tested in their analysis. We extend their analysis by simultaneously fitting both the phenotypic and the marital components of the covariance matrix and examining the covariance matrix of the phenotypic and marital parameter estimates.

\section{MODEL DEVELOPMENT}

\section{Predicted Covariance Matrix}

When marital assortment is modeled using conditional paths, the expected covariance matrix for spouses is of the form

$$
\Sigma=\left[\begin{array}{rr}
\mathbf{R}_{\mathrm{H}} & \mathbf{R}_{\mathrm{H}} \mathrm{D}^{\prime} \mathbf{R}_{\mathrm{W}} \\
\mathbf{R}_{\mathrm{W}} \mathrm{DR}_{\mathrm{H}} & \mathbf{R}_{\mathrm{W}}
\end{array}\right],
$$

where $\mathbf{R}_{\mathbf{W}}$ is the phenotypic covariance matrix for wives, $\mathbf{R}_{\mathbf{H}}$ is the phenotypic covariance matrix for husbands, and $\mathbf{D}^{\prime}$ is the matrix of conditional paths with rows and columns corresponding to husband and wife variables, respectively (see Van Eerdewigh, 1982; Carey, 1986). The derivation of these expectations has been described in detail by Fulker (1988); the basis for the conditional path theory lies in the work on selection initiated by Pearson (1902) and generalized 
such as LISREL 7 (Jöreskog and Sörbom, 1988) or COSAN (Fraser, 1988) is not immediately obvious and is not explicitly treated in either user's guide.

\section{Structural Model}

In principle, any structural equation model may be implemented in a computer program in an infinite number of ways. For example, estimation of a variance could be achieved by estimating the $2 n^{\text {th }}$ root of the variance with the matrix product $\mathbf{F}_{\mathbf{1}} \ldots \mathbf{F}_{n} \mathbf{C F}^{\prime}{ }_{n} \ldots \mathbf{F}^{\prime}{ }_{\mathbf{1}}$ for any positive integer $n$. In practice, we wish to use a formulation that is easily understood and simple to implement in any of a number of packages. A further aim may be to limit the size of matrices involved in estimation and to avoid matrix inversion if these factors increase computational demands beyond reasonable limits. In this article we develop formulations for the COSAN and LISREL packages.

By using RAM notation in conjunction with the multivariate generalization of path analysis (McArdle, 1980; McArdle and McDonald, 1984; Volger, 1985), the model for multivariate assortative mating is described succinctly by the path diagram shown in Fig. 1. In this diagram and the following text, the subscripts $\mathbf{H}$ and $\mathbf{W}$ refer to husbands and wives, respectively. In RAM notation the path

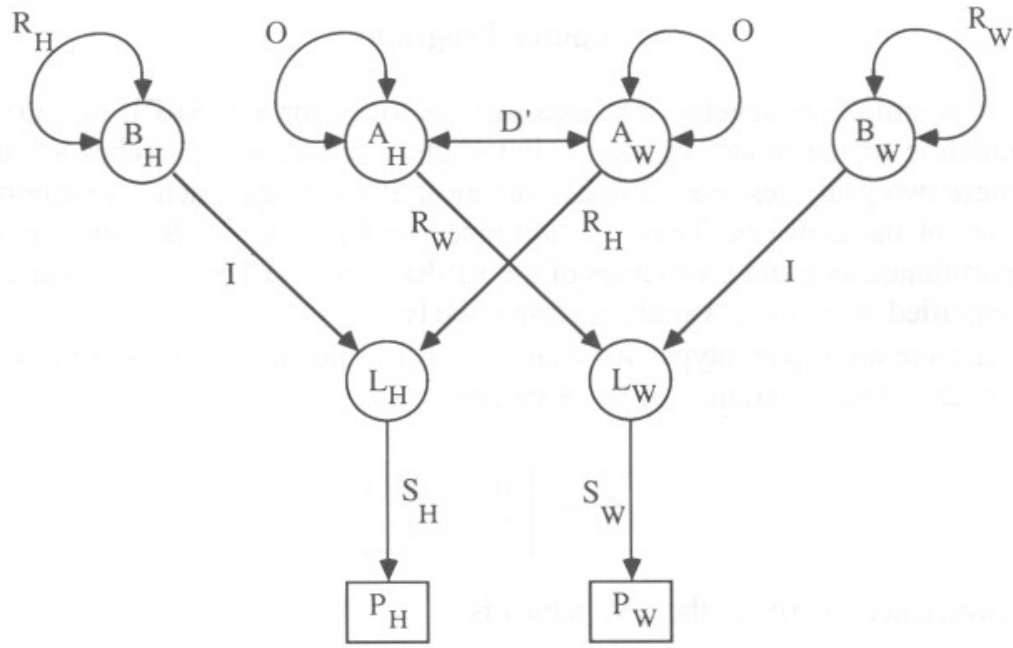

Fig. 1. RAM path diagram of multivariate assortative mating between phenotypes of husbands $\left(P_{\mathrm{H}}\right)$ and wives $\left(P_{\mathrm{w}}\right)$. Latent variables (shown in circles) correspond to standardized phenotypic variables $\left(L_{\mathrm{H}}\right.$ and $\left.L_{\mathrm{w}}\right)$ and their sources of variation $\left(B_{\mathrm{H}}\right.$ and $\left.B_{\mathrm{w}}\right)$ and covariation $\left(A_{\mathrm{H}}\right.$ and $\left.A_{\mathrm{w}}\right)$. Matrices of path coeffecients are as follows: $S$-diagonal matrix of standard deviations; $R$-within-person correlation matrix of phenotypes; $D^{\prime}-$ matrix of delta paths; $I$-identity matrix; $\mathrm{O}$-null matrix. 
analyst is required to draw all variables and model parameters in the diagram. Parameters include single-headed "arrows" for all regression-based causal relations and double-headed "slings" for all covariance-based correlations.

There are three levels of variables in this diagram: ultimate latent variables $\mathbf{A}$ and $\mathbf{B}$ (top row), standardized intermediary variables $\mathbf{L}$ (middle row), and observed variables $\mathbf{P}$ (bottom row). Each variable in the diagram represents a vector of variables, and each of the path coefficients represents a matrix of path coefficients between two such vectors. Of the ultimate variables, there are two basic types, those that have a self-correlation of 1.0 (B variables) and those with a self-correlation of zero (A variables). Ultimate variables without self-correlation do not contribute to the variance of dependent variables; they contribute only to covariance.

The matrices of path coefficients in Fig. 1 are of special form. Those matrices labeled $\mathbf{I}$ are identity matrices, those labeled $\mathbf{O}$ are null matrices, and those labeled $\mathbf{R}$ are correlation matrices. The matrices $\mathbf{S}_{\mathbf{H}}$ and $\mathbf{S}_{\mathbf{W}}$ are diagonal matrices containing the standard deviations of husbands' and wives' variables, respectively. The matrix $\mathbf{D}^{\prime}$ is the matrix of standardized copaths between husband variables (corresponding to columns) and wife variables (corresponding to rows)

\section{Computer Programs}

It is simple to develop the necessary matrices for a LISREL or COSAN formulation of the model in Fig. 1, but slightly different approaches are used for these two packages. For LISREL, the most efficient approach is to minimize the size of the matrices. Thus, the ultimate variables (A and $\mathbf{B}$ ), respectively, are partitioned as $\xi$ and $\zeta$ variables of the LISREL model. The $\mathbf{L}$ and $\mathbf{P}$ variables are specified as $\eta$ and $y$ variables, respectively.

If there are $n$ phenotypes for analysis, all component matrices are of order $(2 n \times 2 n)$. The covariance of the $\xi$ variables is

$$
\boldsymbol{\Phi}=\left[\begin{array}{cc}
\mathbf{0}_{n} & \mathbf{D}^{\prime} \\
\mathbf{D}_{n} & \mathbf{0}_{n}
\end{array}\right],
$$

the covariance matrix of the $\zeta$ variables is

$$
\Psi=\left[\begin{array}{cc}
\mathbf{R}_{\mathbf{H}} & \mathbf{0}_{n} \\
\mathbf{0}_{n} & \mathbf{R}_{\mathbf{W}}
\end{array}\right],
$$


in LISREL and is equal to the $\boldsymbol{\psi}$ matrix above. The regression of the $y$ variables on the $\eta$ variables is

$$
\Lambda_{y}=\left[\begin{array}{cc}
\mathbf{S}_{\mathbf{H}} & \mathbf{0}_{n} \\
\mathbf{0}_{n} & \mathbf{S}_{\mathbf{W}}
\end{array}\right],
$$

where $\mathbf{S}_{\mathbf{H}}$ and $\mathbf{S}_{\mathbf{W}}$ are diagonal matrices of the standard deviations of the husbands and wives respectively.

In COSAN, models are specified as $\mathbf{F}_{\mathbf{1}} \ldots . \mathbf{F}_{n} \mathbf{C F}_{n}{ }_{n} \ldots . \mathbf{F}^{\prime}{ }_{1}$, where the matrices $\mathbf{F}_{\mathrm{i}}$ may be inverses if required. In order to use the above formulation, the partition between the $\mathbf{A}$ and $\mathbf{B}$ variables has to be removed, so that the covariance matrix of ultimate variables is

$$
\mathbf{C}=\left[\begin{array}{cccc}
\mathbf{O}_{n} & \mathbf{D}^{\prime}{ }_{n} & \mathbf{0}_{n} & \mathbf{0}_{n} \\
\mathbf{D}_{n} & \mathbf{0}_{n} & \mathbf{0}_{n} & \mathbf{0}_{n} \\
\mathbf{0}_{n} & \mathbf{0}_{n} & \mathbf{R}_{\mathbf{H}} & \mathbf{0}_{n} \\
\mathbf{0}_{n} & \mathbf{0}_{n} & \mathbf{0}_{n} & \mathbf{R}_{\mathbf{W}}
\end{array}\right],
$$

where $\mathbf{O}_{n}$ and $\mathbf{D}_{n}$ are the null and conditional path matrices, respectively, that are each of order $n$. Note that the vectors of ultimate variables have been ordered alphabetically for this treatment. The matrix $\mathbf{F}_{2}$ corresponds to the matrices of paths from the ultimate variables to the standardized intermediary variables in Fig. 1. This matrix is of order $(2 n \times 2 n)$ and is of the form

$$
\mathbf{F}_{\mathbf{2}}=\left[\begin{array}{cccc}
\mathbf{R}_{\mathbf{H}} & \mathbf{0}_{n} & \mathbf{I}_{n} & \mathbf{0}_{n} \\
\mathbf{0}_{n} & \mathbf{R}_{\mathbf{W}} & \mathbf{0}_{n} & \mathbf{I}_{n}
\end{array}\right] .
$$

The matrix $F_{1}$ is of order $(2 n \times 2 n)$ and is of the form

$$
\mathbf{F}_{1}=\left[\begin{array}{cc}
\mathbf{S}_{\mathbf{H}} & \mathbf{0}_{n} \\
\mathbf{0}_{n} & \mathbf{S}_{\mathbf{W}}
\end{array}\right] \text {. }
$$

For the COSAN approach, the correspondence between diagram and program specification is clearer. Each matrix has been formed directly from the diagram by assigning columns of the matrix to variables that are causal and rows of the matrix to variables that are being caused. For example, the columns of the $\mathbf{F}_{2}$ matrix correspond to the vectors $\mathbf{A}_{\mathbf{H}}, \mathbf{A}_{\mathbf{W}}, \mathbf{B}_{\mathbf{H}}$, and $\mathbf{B}_{\mathbf{W}}$ in the top row of the diagram, and the rows correspond to the vectors of standardized intermediary variables $\mathbf{L}_{\mathbf{H}}$ and $\mathbf{L}_{\mathbf{W}}$.

In LISREL 7, it is necessary to set the admissibility test off ( $\mathrm{AD}=\mathrm{OFF})$ on the OU card, since the matrix $\boldsymbol{\Phi}$ is nonpositive definite by definition. The full LISREL 7 input file is shown in Appendix I. The shorter COSAN II input file is shown in Appendix II. 


\section{REANALYSIS OF IQ AND PERSONALITY DATA}

The data reported by Phillips et al. (1988) were reanalyzed in order to examine the consequences of assuming zero correlation between estimates of the phenotypic and marital covariances. The variance-covariance matrix part of the COSAN input file is shown in Appendix II. Although seven places of decimals were used for analysis, only four places are shown in the Appendix to save space.

The full model has 78 parameters, and space prevents publication of the $(78 \times 78)$ matrix of correlations between parameter estimates. There is little reason to present the matrix since almost all of the correlations between phenotypic parameters and marital parameters are less than .01 for these data. In addition, Neale et al. (1990) show that standard errors of parameter estimates are not invariant to transformation, and the same applies to covariances between parameter estimates. However, the near-complete independence of the two sets of parameters is reflected in the goodness of fit statistics, which are very close to the values obtained by fitting with fixed phenotypic variance covariance matrices. The two sets of $\chi^{2}$ statistics are shown in Table I.

\section{DISCUSSION}

LISREL and COSAN implementations of the assortative mating model of Carey (1986) have been described. The RAM notation of McArdle (1980) is combined with the multivariate extension of path analysis described by McArdle (1980), McArdle and McDonald (1984), and Vogler (1985). We hope that the LISREL formulation will assist others to explore primary and secondary assortative mating in other variables. Needed extensions of the method include the

Table I. Fit Statistics for a Variety of Submodels of a Multivariate Model of Phenotypic Assortment with Phenotypic Matrices as Free vs. Fixed Parameters

\begin{tabular}{llll}
\hline \multicolumn{1}{c}{ Submodel } & \multicolumn{2}{c}{$\chi^{2}$} & \\
\cline { 2 - 3 } & Fixed & Free & df \\
\hline 1. Symmetry of D & 20.09 & 20.07 & 15 \\
2. (1) and no cross-domain mating & & 27.41 & 23 \\
3. Diagonal D & 27.42 & 41.52 & 30 \\
4. (2) and no g-educn. cross mating & 27.76 & 27.74 & 24 \\
5. (4) and no $g$ assortment & 32.10 & 32.09 & 25 \\
6. (4) and no educn. assortment & 85.27 & 85.26 & 25 \\
\hline
\end{tabular}

${ }^{a}$ The two domains are cognitive (Education and IQ) and Personality (Extraversion, Anxietv. Tough 
treatment of assorative mating in twins and their spouses, to allow resolution of phenotypic and social homogamy (Heath and Eaves, 1985).

Current limitations of LISREL, which lacks any means of specifying constraints, and of COSAN, which does not allow for multiple groups, prevent the development of a number of interesting alternative models of mate selection. These models include mixed social and genetic homogamy, social homogamy based on parental phenotypes, and mixed genetic and cultural transmission.

Relaxation of the simplifying assumptions used by Phillips et al. did not substantively change their results because the correlations between parameter estimates were so small. We note that phenotypic assortment for IQ is considerably smaller than the assortment for the education measure. In addition, since education was measured on a discontinuous scale, the true correlation for educational attainment is likely to have been underestimated [compare polychoric and product-moment correlations between spouses for education given by Heath et al. (1985)]. Only slight attenuation is expected for the (polyserial) correlation between the continuous IQ measure and education. If the true marital correlation for education between spouses is .48 rather than the calculated .43 , then the evidence for direct marital assortment for IQ in the CAP data disappears. The marital correlation for IQ may simply be a secondary correlation due to the correlation with educational attainment for these data.

There appears to be assortative mating across the four personality measures, not simply within measures. However, the different structures of the phenotypic covariance matrices in males and females make the cross assortment difficult to interpret. It is possible that there is no cross assortment in the true sense, but rather that, to some degree, Scale A measures character A in males, yet measures character B in females. Thus apparent cross assortment between Scale A and Scale B may just reflect within-domain assortment. A general model allowing assortative mating between latent variables is discussed elsewhere (Neale, 1989; Neale et al., 1990).

\section{ACKNOWLEDGMENTS}

Dr. K. Phillips kindly provided variance-covariance matrices for reanalysis. The authors are grateful for helpful comments on the manuscript provided by G. Carey, L. J. Eaves, J. K. Hewitt, A. C. Heath, J. M. Meyer, K. Phillips, and C. A. Prescott. 


\section{Appendix I}

LISREL program for fitting a model of multivariate phenotypic assortative mating to data from husbands and wives. The $\mathrm{MO}$ card has been split over two lines; concatenation of these lines is necessary for LISREL input.

PHILLIPS ET AL 1988 FINAL MODEL

DA NG $=1 \mathrm{NI}=12 \mathrm{NO}=334$

LA

'HGFAC' 'HEDUC' 'HEXTR' 'HANX' 'HTOUGH' 'HIND'

'WGFAC' 'WEDUC' 'WEXTR' 'WANX' 'WTOUGW' 'WIND'

$\mathrm{CM}$ fi=casmat.dat

MO NY=12 NX=0 NE=12 NK=12 GA=FU,FI

$\mathrm{PH}=\mathrm{SY}, \mathrm{FI} \mathrm{PS}=\mathrm{SY}, \mathrm{FI} \mathrm{TE}=\mathrm{ZE}$

LE

*

'HGFAC' 'HEDUC' 'HEXTR' 'HANX' 'HTOUGH' 'HIND'

'WGFAC' 'WEDUC' 'WEXTR' 'WANX' 'WTOUGW' 'WIND'

LK

$*$

'HGFAC' 'HEDUC' 'HEXTR' 'HANX' 'HTOUGH' 'HIND'

'WGFAC' 'WEDUC' 'WEXTR' 'WANX' 'WTOUGW' 'WIND'

PA GA

$*$

$6^{*} 16^{*} 06^{*} 16^{*} 06^{*} 16^{*} 06^{*} 16^{*} 06^{*} 16^{*} 06^{*} 16^{*} 0$ $6 * 06 * 16 * 06 * 16 * 06 * 16 * 06 * 16 * 06 * 16 * 06 * 1$ PA PH

*

0

00

$\begin{array}{lllll}0 & 0 & 0\end{array}$

$\begin{array}{lllllllllllllllll}0 & 0 & 0 & 0\end{array}$

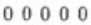

00000000

100000000

01100000000

000111110000

0011111100000

00111110000000

$\begin{array}{llllllllllll}0 & 0 & 1 & 1 & 1 & 1 & 0 & 0 & 0 & 0 & 0 & 0\end{array}$

PA PS

*

$21^{*} 16^{*} 01^{*} 6^{*} 02^{*} 16^{*} 03^{*} 16^{*} 04^{*} 16^{*} 05^{*} 16^{*} 06^{*} 1$

EQ GA(2,1) GA(1,2) PS(2,1) PS(1,2)

EQ GA(3,1) GA(1,3) PS $(3,1)$ PS $(1,3)$

EQ GA(4,1) GA(1,4) PS(4,1) PS $(1,4)$

EQ GA(5,1) GA(1,5) PS(5,1) PS $(1,5)$

EQ GA(6,1) GA(1,6) PS(6,1) PS $(1,6)$

$\mathrm{EQ} \mathrm{GA}(7,1)$ GA(1,7) PS(7,1) PS $(1,7)$

EQ GA( 8,1$)$ GA(1,8) PS $(8,1)$ PS $(1,8)$

EQ GA(9,1) GA(1,9) PS $(9,1)$ PS $(1,9)$

EQ GA $(10,1)$ GA $(1,10)$ PS $(10,1)$ PS $(1,10)$
EQ GA(11,1) GA(1,11) PS(11,1) PS(1,11)

EQ GA(12,1) GA(1,12) PS(12,1) PS(1,12)

EQ GA $(3,2)$ GA(2,3) PS $(3,2)$ PS $(2,3)$

EQ GA(4,2) GA(2,4) PS $(4,2)$ PS $(2,4)$

EQ GA $(5,2)$ GA(2,5) PS $(5,2)$ PS $(2,5)$

EQ GA(6,2) GA(2,6) PS $(6,2)$ PS $(2,6)$

EQ GA $(7,2)$ GA $(2,7)$ PS( 7,2$)$ PS $(2,7)$

EQ GA $(8,2)$ GA $(2,8)$ PS $(8,2)$ PS $(2,8)$

EQ GA $(9,2)$ GA $(2,9)$ PS $(9,2)$ PS $(2,9)$

EQ GA(10,2) GA(2,10) PS $(10,2)$ PS $(2,10)$

EQ GA(11,2) GA(2,11) PS(11,2) PS $(2,11)$

EQ GA(12,2) GA(2,12) PS(12,2) PS $(2,12)$

EQ GA $(4,3)$ GA $(3,4)$ PS $(4,3)$ PS $(3,4)$

EQ GA(5,3) GA(3,5) PS $(5,3)$ PS $(3,5)$

EQ GA $(6,3)$ GA $(3,6)$ PS $(6,3)$ PS $(3,6)$

EQ GA( 7,3$)$ GA $(3,7)$ PS $(7,3)$ PS $(3,7)$

EQ GA $(8,3)$ GA $(3,8)$ PS $(8,3)$ PS $(3,8)$

EQ GA $(9,3)$ GA $(3,9)$ PS $(9,3)$ PS $(3,9)$

EQ GA(10,3) GA(3,10) PS $(10,3)$ PS $(3,10)$

EQ GA(11,3) GA(3,11) PS(11,3) PS $(3,11)$

EQ GA(12,3) GA(3,12) PS(12,3) PS $(3,12)$

EQ GA $(5,4)$ GA $(4,5)$ PS $(5,4)$ PS $(4,5)$

EQ GA $(6,4)$ GA(4,6) PS $(6,4)$ PS $(4,6)$

EQ GA $(7,4)$ GA $(4,7)$ PS $(7,4)$ PS $(4,7)$

EQ GA $(8,4)$ GA $(4,8)$ PS $(8,4)$ PS $(4,8)$

EQ GA $(9,4)$ GA $(4,9)$ PS $(9,4)$ PS $(4,9)$

EQ GA(10,4) GA(4,10) PS(10,4) PS $(4,10)$

EQ GA(11,4) GA(4,11) PS $(11,4)$ PS $(4,11)$

EQ GA $(12,4)$ GA $(4,12)$ PS(12,4) PS $(4,12)$

EQ GA $(6,5)$ GA $(5,6)$ PS $(6,5)$ PS $(5,6)$

EQ GA $(7,5)$ GA $(5,7)$ PS $(7,5)$ PS $(5,7)$

EQ GA $(8,5)$ GA $(5,8)$ PS $(8,5)$ PS $(5,8)$

EQ GA $(9,5)$ GA $(5,9)$ PS $(9,5)$ PS $(5,9)$

EQ GA $(10,5)$ GA $(5,10)$ PS $(10,5)$ PS $(5,10)$

EQ GA(11,5) GA(5,11) PS $(11,5)$ PS $(5,11)$

EQ GA(12,5) GA $(5,12)$ PS $(12,5)$ PS $(5,12)$

EQ GA $(7,6)$ GA $(6,7)$ PS $(7,6)$ PS $(6,7)$

EQ GA( 8,6$)$ GA(6,8) PS $(8,6)$ PS $(6,8)$

EQ GA $(9,6)$ GA $(6,9)$ PS $(9,6)$ PS $(6,9)$

EQ GA(10,6) GA $(6,10)$ PS $(10,6)$ PS $(6,10)$

EQ GA(11,6) GA(6,11) PS $(11,6)$ PS $(6,11)$

EQ GA(12,6) GA $(6,12)$ PS $(12,6)$ PS $(6,12)$

EQ GA $(8,7)$ GA $(7,8)$ PS $(8,7)$ PS $(7,8)$

EQ GA $(9,7)$ GA $(7,9)$ PS $(9,7)$ PS $(7,9)$

EQ GA $(10,7)$ GA $(7,10)$ PS $(10,7)$ PS $(7,10)$

EQ GA(11,7) GA $(7,11)$ PS $(11,7)$ PS $(7,11)$

EQ GA(12,7) GA $(7,12)$ PS $(12,7)$ PS $(7,12)$

EQ GA $(9,8)$ GA $(8,9) \operatorname{PS}(9,8) \operatorname{PS}(8,9)$

EQ GA $(10,8)$ GA $(8,10)$ PS $(10,8) \operatorname{PS}(8,10)$

EQ GA(11,8) GA(8,11) PS(11,8) PS $(8,11)$

EQ GA(12,8) GA(8,12) PS $(12,8) \operatorname{PS}(8,12)$

EQ GA $(10,9)$ GA $(9,10)$ PS $(10,9)$ PS $(9,10)$

EQ GA(11,9) GA $(9,11)$ PS $(11,9)$ PS $(9,11)$

EQ GA(12,9) GA $(9,12)$ PS(12,9) PS $(9,12)$

EQ GA $(11,10)$ GA $(10,11)$ PS $(11,10) \operatorname{PS}(10,11)$

EQ GA(12,10) GA(10,12) PS $(12,10)$ PS $(10,12)$

$\mathrm{EQ} \mathrm{GA}(12,11) \mathrm{GA}(11,12)$ PS(12,11) PS $(11,12)$

EQ PH $(8,1)$ PH $(7,2)$

EQ $\mathrm{PH}(9,1) \mathrm{PH}(7,3)$

$\mathrm{EQ} \mathrm{PH}(10,1) \mathrm{PH}(7,4)$ 
$\mathrm{EQ} r \mathrm{n}(12,3) \mathrm{rn}(11,0)$

FR LY (1,1) LY(2,2) LY (3,3) LY(4,4) LY $(5,5) \operatorname{LY}(6,6)$

FR LY $(7,7)$ LY $(8,8)$ LY $(9,9)$ LY $(10,10)$

FR LY $(11,11)$ LY $(12,12)$

FI GA(1,1) GA(2,2) GA(3,3) GA(4,4)

FI GA(5,5) GA(6,6) GA(7,7) GA(8,8)

FI GA(9,9) GA(10,10) GA(11,11) GA(12,12)

FI PS(1,1) PS(2,2) PS(3,3) PS $(4,4)$

FI PS $(5,5)$ PS $(6,6)$ PS $(7,7)$ PS $(8,8)$

FI PS $(9,9)$ PS $(10,10)$ PS $(11,11)$ PS $(12,12)$

VA 1.0 GA(1,1) GA(2,2) $\mathrm{GA}(3,3) \mathrm{GA}(4,4)$

VA $1.0 \mathrm{GA}(5,5) \mathrm{GA}(6,6) \mathrm{GA}(7,7) \mathrm{GA}(8,8)$

VA $1.0 \mathrm{GA}(9,9) \mathrm{GA}(10,10) \mathrm{GA}(11,11) \mathrm{GA}(12,12)$

VA 1.0 PS(1,1) PS(2,2) PS $(3,3) \operatorname{PS}(4,4)$

VA 1.0 PS(5,5) PS(6,6) PS $(7,7)$ PS $(8,8)$

VA $1.0 \operatorname{PS}(9,9) \operatorname{PS}(10,10) \operatorname{PS}(11,11) \operatorname{PS}(12,12)$

VA 0.0 PH(1,1) $\mathrm{PH}(2,2) \mathrm{PH}(3,3) \mathrm{PH}(4,4)$

VA 0.0 PH(5,5) $\mathrm{PH}(6,6) \mathrm{PH}(7,7) \mathrm{PH}(8,8)$

VA 0.0 PH $(9,9) \mathrm{PH}(10,10) \mathrm{PH}(11,11) \mathrm{PH}(12,12)$

ST .1 ALL

ST 2.0 LY (1,1)-LY $(12,12)$

OU NS AD=OFF SO RS PC

\section{Appendix II}

COSAN input file fitting a model of multivariate phenotypic assortative mating to data from husbands and wives.

PHILLIPS ET AL 1988 FINAL MODEL

$\mathrm{DEC}=6, \mathrm{ROWS}=12, \mathrm{LARGEST}=24, \mathrm{ORDER}=2, \mathrm{C}$

$\mathrm{EST}=\mathrm{LS}+\mathrm{ML}, \mathrm{NO}=334, \mathrm{ACC}=.000001, \mathrm{C}$

IN $=$ LSYM, MAX $=500$, VALUE $=.0$

(12D10.7)

0.8195

0.74074 .0259

0.01540 .56943 .6999

$-0.0547-0.2443-0.06213 .1069$

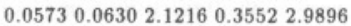

$\begin{array}{lllllll}0.2090 & 0.7767 & 0.8328 & -0.3515 & 0.9064 & 2.6083\end{array}$

$\begin{array}{lllllll}0.1779 & 0.4905 & 0.0743 & -0.0539 & 0.1146 & 0.3566\end{array}$

1.0151

$\begin{array}{llllll}0.3758 & 2.0013 & 0.7029 & -0.2894 & 0.1197 & 0.8569\end{array}$

1.06995 .4494

$\begin{array}{llllll}0.1396 & 0.1702 & 0.8243 & -0.1581 & 0.3468 & 0.1340\end{array}$

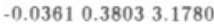

$0.0505-0.2281-0.13360 .26730 .0626-0.0436$

$-0.1708-0.5290-0.80562 .6163$

$\begin{array}{llllll}0.1657 & 0.0294 & 0.0254 & -0.0632 & 0.1553 & 0.2716\end{array}$
ROW $3=14180222324 \quad 18^{*} 0$

ROW $4=1519220252618 * 0$

ROW $5=1620232502718^{*} 0$

ROW $6=1721242627018^{*} 0$

ROW $7=6^{*} 0 \quad 0 \quad 2829303132 \quad 12^{*} 0$

ROW $8=6^{*} 02803334353612 * 0$

ROW $9=6^{*} 02933037383912 * 0$

ROW $10=6 *^{*} 030343704041 \quad 12 * 0$

ROW $11=6^{*} 03135384004212^{*} 0$

ROW $12=6^{*} 03236394142012 * 0$

INITIAL

$\operatorname{DIAG}(1,1)$ TO $(12,12)=12 * 1$

$\operatorname{DIAG}(1,13)$ TO $(12,24)=12 * 1$

MATRIX P 24 BY 24

PATTERN

ROW $1=0$

ROW $2=2 * 0$

ROW $3=3^{*} 0$

ROW $4=4^{*} 0$

ROW $5=5^{*} 0$

ROW $6=6{ }^{*} 0$

ROW $7=4344454647480$

ROW $8=4950515253542^{*} 0$

ROW $9=5556575859603^{*} 0$

ROW $10=6162636465664^{*} 0$

ROW $11=6768697071725^{*} 0$

ROW $12=7374757677786^{*} 0$

ROW $13=12 * 00$

ROW $14=12 * 0130$

ROW $15=12 * 014180$

ROW $16=12 * 01519220$

ROW $17=12 * 0162023250$

ROW $18=12 * 017212426270$

ROW $19=18^{*} 00$

ROW $20=18 * 0280$

ROW $21=18 * 029330$

ROW $22=18 * 03034370$

ROW $23=18^{*} 0313538400$

ROW $24=18^{*} 032363941420$

INITIAL

DIAG $(13,13)$ TO $(24,24)=12 * 1$

BEGIN 


\section{REFERENCES}

Aitken, A. C. (1934). Note on selection from a multivariate normal population. Proc. Edin. Math. Soc. B 4:106-110.

Carey, G. (1986). A general multivariate approach to linear modeling in human genetics. Am. J. Hum. Genet. 39:775-786.

Cattell, R. B., Eber, H. W., and Tatsuoka, M. (1970). Handbook for the Sixteen Personality Factor Questionnaire (16PF), Institute for Personality and Ability Testing, Champaign, Ill.

Cloninger, C. R. (1980). Interpretation of intrinsic and extrinsic structural relations by path analysis: Theory and applications to assortative mating Genet. Res. 36:133-145.

Fraser, C. (1988). COSAN User's Guide, Unpublished documentation, Centre for Behavioural Studies in Education, University of New England, Armidal, NSW, Australia 2351.

Fulker, D. W. (1988). Genetic and cultural transmission in human behavior. In Weir, B. S., Eisen, E. J., Goodman, M., and Namkoong, G. (eds.), Proceedings of the Second International Conference on Quantitative Genetics Massachusetts, Sinauer Associates, Sunderland, Mass.

Heath, A. C., and Eaves, L. J. (1985). Resolving the effects of phenotype and social background on mate selection. Behav. Genet. 15:15-30.

Heath, A. C., Berg, K., Eaves, L. J., Solaas, M. H., Sundet, J., Nance, W. E., Corey, L. A., and Magnus, P. (1985). No decline in assortative mating for educational level. Behav. Genet. 15:349-369.

Jöreskog, K. G., and Sörbom, D. (1988). LISREL 7: A Guide to the Program and Applications, SPSS, Chicago.

McArdle, J. J. (1980). Causal modeling applied to psychonomic systems simulation. Behav. Res. Methods Implement. 12:193-209.

McArdle, J. J., and Horn, J. L. (1989). An effective graphic representation for linear structural equation models. Multivar. Behav. Res. (in press).

McArdle, J. J., and McDonald, R. P. (1984). Some algebraic properties of the reticular action model for moment structures. Br. J. Math. Stat. Psychol. 37:234-251.

Neale, M. C. (1989). A latent variable model of assortative mating. Behav. Genet. 19:770 (abstract).

Neale, M. C., Eaves, L. J., Heath, A. C., and Hewitt, J. K. (1990). Confirmatiory canonical correlation analysis: A parsimonious model for assortative mating (in preparation).

Pearson, K. (1902). Mathematical contributions to the theory of evolution. XI. On the influence of natural selection on the variability and correlation of organs. Phil. Trans. R. Soc. Lond. A 200:1-66.

Pearson, K., and Lee, A. (1903). On the laws of inheritance in man. Biometrika 2:357-396.

Phillips, K., Fulker, D. W., Carey, G., and Nagoshi, C. T. (1988). Direct marital assortment for cognitive and personality variables. Behav. Genet. 18:347-356.

Van Eerdewegh, P. (1982). Statistical Selection in Multivariate Systems with Applications in Quantitative Genetics, Unpublished doctoral dissertation, Washington University, St. Louis, Mo.

Vogler, G. P. (1985). Multivariate path analysis of familial resemblance. Genet. Epid. 2:35-54.

Edited by David W. Fulker 